\title{
Towards a High-Accuracy RF Wien Filter for Spin Manipulation at COSY Jülich
}

\author{
Jamal Slim* and Dirk Heberling ${ }^{\dagger}$ \\ Institute of High Frequency Technology, RWTH Aachen University \\ E-mail: slimeihf.rwth-aachen.de
}

\begin{abstract}
The JEDI collaboration is aiming for electric dipole moment (EDM) measurements of deuterons and protons at COSY-Jülich. A high accuracy RF Wien filter, operating at the spin harmonic frequencies is planned to be integrated in the COSY ring at the Forschungszentrum Jülich. This RF Wien filter is intended for spin tune modulation purposes allowing to perform EDM measurements. Because of the smallness of the EDM signal, critical requirements on this Wien filter have been imposed both in terms of field homogeneity and orthogonality. The transverse electromagnetic (TEM) mode of a parallel-plates waveguide fulfills the requirements. This work will show both the electrodynamics analysis together with the engineering design.
\end{abstract}

XVIth International Workshop in Polarized Sources, Targets, and Polarimetry, PSTP2015,

14-18 September 2015,

Bochum, Germany

${ }^{*}$ Speaker.
${ }^{\dagger}$ For the JEDI collaboration 


\section{Introduction}

The generalized Thomas-BMT equation including the EDM term [1, 2] describes the spin dynamics in electromagnetic storage rings with $\vec{\beta} \cdot \vec{E}=\vec{\beta} \cdot \vec{B}=0$; neglecting the longitudinal field contributions, the T-BMT equation reads:

$$
\begin{aligned}
\frac{d \vec{S}}{d t} & =\vec{S} \times\left(\vec{\Omega}_{M D M}+\vec{\Omega}_{E D M}\right) \\
\vec{\Omega}_{M D M} & =\frac{q}{m}\left((1+\gamma G) \vec{B}-\left(\gamma G+\frac{\gamma}{\gamma+1}\right) \vec{\beta} \times \frac{\vec{E}}{c}\right) \\
\vec{\Omega}_{M D M} & =\frac{q}{m} \frac{\eta}{2}\left(\frac{\vec{E}}{c}+\vec{\beta} \times \vec{B}\right)
\end{aligned}
$$

where, $\vec{E}$ and $\vec{B}$ are the electric field and magnetic flux in the laboratory system, $\vec{S}$ the spin vector, $\vec{\Omega}$ the angular velocity of the spin precession, $G$ the anomalous magnetic moment ( $G=-0.143$ for deuterons [3]). The dimensionless factor $\eta$ describes the strength of the particle's permanent EDM relative to its MDM (Magnetic Dipole Moment), $t$ is the time in the laboratory system, $q$ is the elementary charge, $m=\gamma \cdot m_{0}$ is the relativistic mass of the particle ( $m_{0}$ is the rest mass), $\beta$ and $\gamma$ are the relativistic Lorentz factors. The Standard Model (SM) predicts $\eta$ in the range of $10^{-15}$ which is not possible to be measured with the current technologies. One proposed method to perform an EDM measurement in a magnetic storage ring is the use of an induced spin resonance. This resonance is created artificially by the application of a radio frequency radial electric and vertical magnetic field in the standard Wien filter configuration. Consequently, additional effects arising from betatron oscillations are minimized, while an EDM related vertical polarization buildup serves as an observable. The details of this method can be found in [4].

This paper describes the electromagnetic design and simulation of a high precision RF Wien filter. Section 2 presents the proof-of-concept of using the parallel-plates waveguide as an RF Wien filter. The electrodynamics in addition to some simulations are shown. Section 3 describes some design and simulation aspects of the fully detailed system. Section 4 summarizes the results.

\section{Electrodynamics Analysis}

The transverse electromagnetic (TEM) mode is a solution of Maxwell equations with the electric and magnetic fields being orthogonal in the transverse plane and with no field components in the direction of propagation. This mode can be used to represent the fields inside the intended RF Wien filter. The parallel-plates waveguide (as being two-conductor system) is a structure that supports the TEM mode and it is adequate to be used as a Wien filter at RF frequency as will be shown later.The basic geometry of the parallel-plates waveguide is shown in figure 1 . To have a pure TEM mode is nearly difficult in practice (see [5]); for example, the length $l$ must extend to infinity and $w \gg d$ ( $w$ is the width of the electrodes and $d$ is their separation distance). In reality, the system will be truncated and the solution will deviate slightly from the ideal case. The link between the TEM mode and the Wien filter condition is in the field quotient i.e. the ratio between 


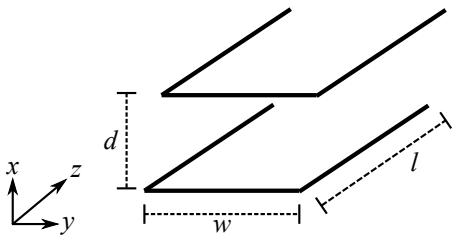

(a) basic geometry

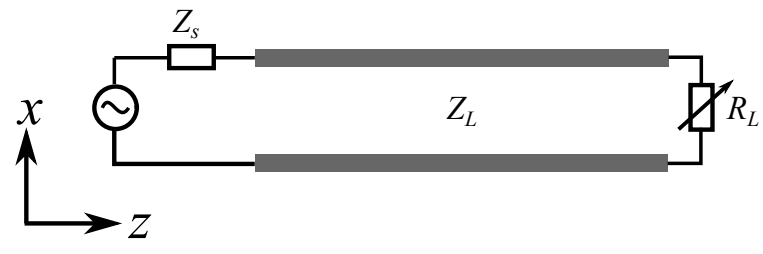

(b) circuit

Figure 1: Proposed setup of the RF Wien filter

the electric and magnetic field. For deuterons at $970 \mathrm{MeV} / \mathrm{c}(\beta=0.459)$ the required field quotient is around $173 \Omega$. Having a predefined geometry, the field quotient is constant (the matched case). To adapt the field quotient and fulfill the Wien filter condition, the wave mismatch theory is going to be used. Only the end solution is considered. In the purely forward wave propagation case, the load impedance is perfectly matched to the generator impedance as can be seen in the return loss in figure 2(a).

$$
\begin{aligned}
\vec{E}(x, y, z) & =E_{0} \vec{e}_{x} e^{-j k z} \\
\vec{H}(x, y, z) & =E_{0} \frac{1}{Z_{w}} \vec{e}_{y} e^{-j k z}=H_{0} \vec{e}_{y} e^{-j k z}
\end{aligned}
$$

$E_{0}$ is the amplitude of the electric field, $k$ is the wavenumber, $z$ is the direction of propagation and $Z_{w}$ is the wave impedance. For verification purposes, a full-wave simulation of the simple geometry has been conducted. The electric and magnetic fields are directed in the $\vec{e}_{x}$ and $\vec{e}_{y}$ directions respectively. With $0.5 \mathrm{~W}$ as input power with $w=182 \mathrm{~mm}, d=10 \mathrm{~mm}, l=700 \mathrm{~mm}$, source impedance $Z_{s}=50 \Omega$ and the load impedance $R_{L}=57.5 \Omega$, the fully matched case has been achieved. The reflected wave has a magnitude less than $-50 \mathrm{~dB}$. The magnitude of the electric $E_{0}$ and magnetic $H_{0}$ fields read $99.75 \pm 0.01 \mathrm{~V} / \mathrm{m}$ and $0.2794 \pm 0.0001 \mathrm{~A} / \mathrm{m}$ respectively (figure 3(a) and figure 3(b)). The wave impedance is in this case around $356 \Omega$ (figure 3(c)). The corresponding Lorentz force at the center is $51.91 \pm 0.01 \mathrm{eV} / \mathrm{m}$ (figure 3(d)). Under the current case, the Wien filter condition is not fulfilled. The field quotient must be reduced to $173 \Omega$ which corresponds to a zero value of the Lorentz force. To solve this problem, the electric field must be reduced and the magnetic field must be increased in a way that the field quotient converges to $173 \Omega$. The key is to introduce a reflection back into the structure so that the reflected electric field is in the $-\vec{e}_{x}$ and the reflected magnetic field is the $\vec{e}_{y}$ direction. The reflection is controlled by changing the value of the load resistance. Looking at figure 2(a), the return loss has increased to $-31 \mathrm{~dB}$. (This value is still good enough to ensure a good performance of the system). In the case of wave mismatch, the reflected components must be added to equations 2 as:

$$
\begin{aligned}
\vec{E} & =\vec{E}^{f}+\vec{E}^{b} \\
& =\vec{E}^{f}+\Gamma \vec{E}^{f} \\
& =\vec{E}^{f}(1+\Gamma) \\
& =\vec{E}^{f}\left(1+\frac{R_{L}-Z_{L}}{R_{l}+Z_{L}}\right)
\end{aligned}
$$

Here, $\Gamma$ is the reflection coefficient, $R_{L}$ represents the value of the load resistance (pure resistive 


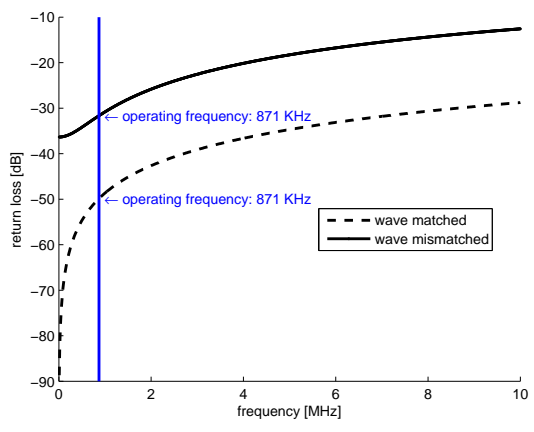

(a) The return loss of the system in $\mathrm{dB}$.

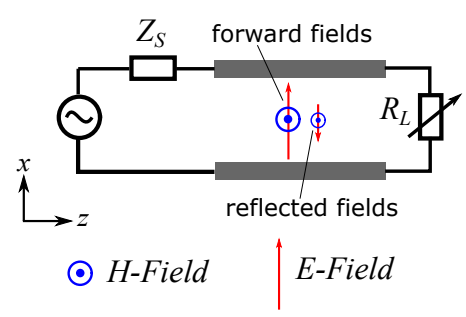

(b) Schematic representation of fields in the structure.

Figure 2: In the matched wave case, only forward waves propagate. Matched means no reflections. In the wave mismatched case: wave reflections are introduced to the structure. The strength of the electric and magnetic fields can be controlled by manipulating the value of the load resistor. Tn this case, the reflected electric field is in the opposite direction of the forward propagating one while for the reflected magnetic field, is in the same direction.

case), $\vec{E}^{f}$ and $\vec{E}^{b}$ are the forward and backward propagating electric fields respectively and $Z_{L}$ is the line impedance of the RF Wien filter. Changing $R_{L}$ to $97 \Omega$, (the source impedance $Z_{s}$ must also be changed to $200 \Omega$ for power matching) the value of the electric field reduces to $64.47 \mathrm{~V} / \mathrm{m}$ which is exactly the value shown by the simulat 1ions. The same analysis follows for the magnetic field, field quotient and the Lorentz force. The results are shown in figure 3.

\section{Implementation and Simulation}

The detailed analysis of the simulations and engineering aspects of the system are discussed in [6]. Only preliminary results are shown in this section. The design model of the final system is shown in figure 4. Ferrite material are used to flatten and concentrate the magnetic field. The ferrites increase the value of the magnetic field by a value of $30 \%$. With $1 \mathrm{~kW}$ as input power, the simulated electromagnetic field profiles together with a cut of the Lorentz force are shown in figure 5: The homogeneity is evaluated on an integral basis. $\vec{E}_{p}=\vec{E}_{y}+\vec{E}_{z}$ and $\vec{H}_{p}=\vec{H}_{x}+\vec{H}_{z}$, represent the parasitic (unwanted) components of the electromagnetic field. In literature, these parasitic components are not considered; only the main field (field inside the structure) is taken into account. Even though the main field constitutes the majority of the field, nevertheless the field errors effect will introduce kicks on the particles at the entry and exit points of the RF Wien filter. Because of the smallness of the EDM signal, field errors must be minimized. The present design provides the following homogeneity values:

$$
\begin{aligned}
& \frac{1}{L} \int_{-L / 2}^{L / 2} \frac{E_{p}}{E} d z=5.8 \times 10^{-5}, \\
& \frac{1}{L} \int_{-L / 2}^{L / 2} \frac{H_{p}}{H} d z=1.5 \times 10^{-5} .
\end{aligned}
$$

$L$ is active length of the Wien filter; it starts and ends where the field values are non-zero. In this design, $L=1550 \mathrm{~mm}$. The field homogeneity values are the results after many numerical simulations 


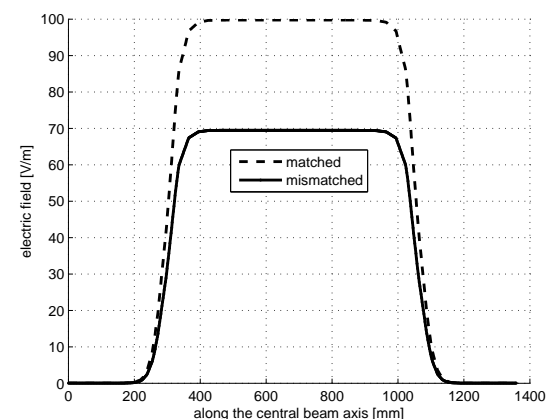

(a) electric field

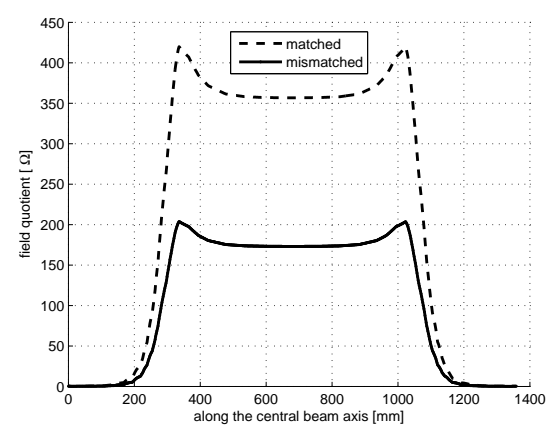

(c) field quotient

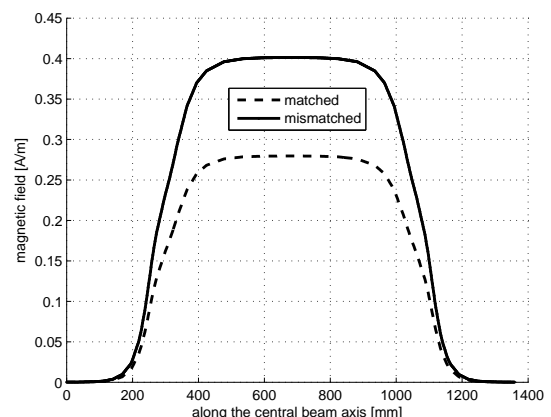

(b) magnetic field

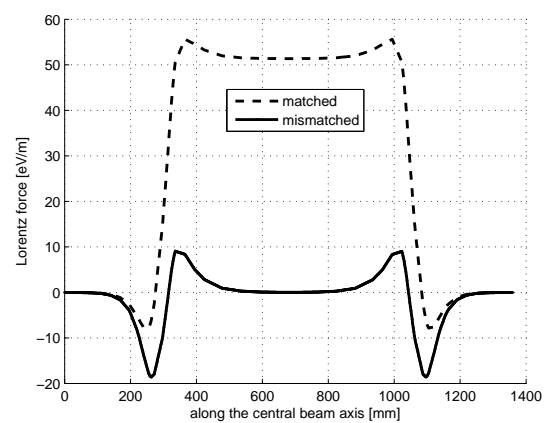

(d) Lorentz force

Figure 3: Proof of concept of the parallel-plates-waveguide as a Wien filter

performed on a graphical processing unit (GPU) cluster. The current distribution was the main factor taken into account. Optimizing the predicted current flow let to such values. The details are described in [6]. For EDM measurements, the beam should not oscillate, and the normalized Lorentz force must be kept near zero. The Lorentz force is the net force exerted on charged particles with applied electric and magnetic fields is defined as:

$$
\int \vec{F}_{\mathrm{L}}(z) d z=\int q(\vec{E}(z)+\vec{v} \times \vec{B}(z)) d z
$$

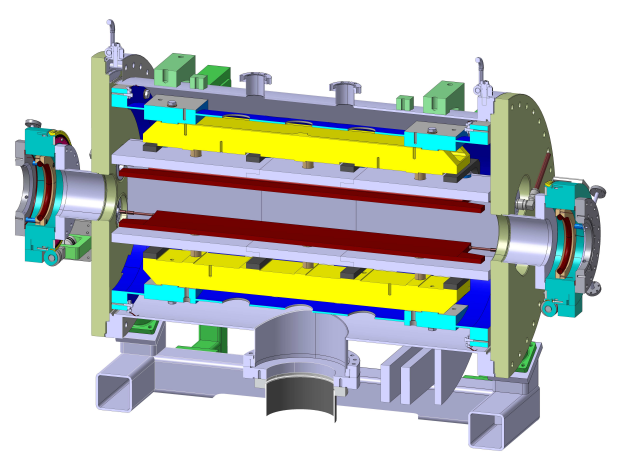

Figure 4: Current design of the RF Wien filter. This is preliminary result. The red part are the electrodes, the lightblue are the ferrites surrounding the electrodes, the yellow elements are the support structure of the electrodes, the grey elements are the clamps holding the ferrite blocks [6] 


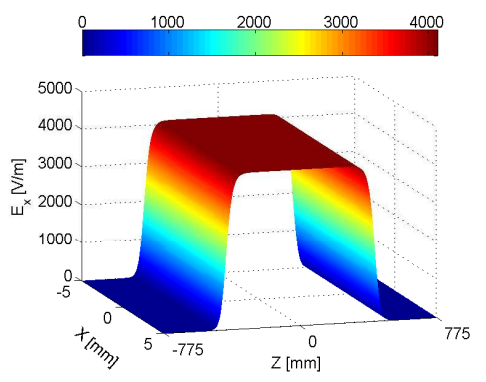

(a) electric field

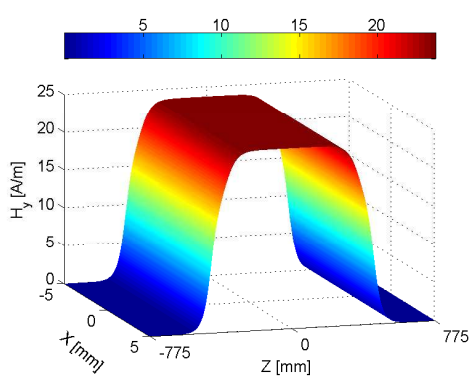

(b) magnetic field

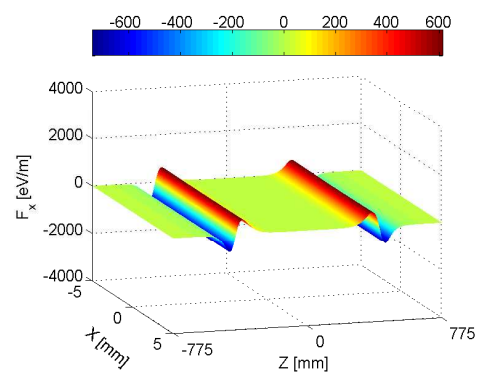

(c) Lorentz force

Figure 5: Preliminary results of the full-wave simulations [6]

$q$ is the elementary charge, $\vec{v}$ is the particles' velocity vector. In our work, the normalized Lorentz force along the beam axis reads:

$$
\frac{1}{L} \int_{-L / 2}^{L / 2} F_{L} d z=1.5 \times 10^{-3} \mathrm{eV} / \mathrm{m},
$$

This value is acceptable because the momentum variation within the beam of $\Delta p / p=10^{-4}$ translates into an acceptable impedance variation of the same order of magnitude. The field quotient, defined as $\frac{E_{x}}{H_{y}}=Z_{0} \beta=377 \cdot \beta_{z} ; \Delta p / p$ represents the longitudinal variation of the particle velocity and consequently the impedance mismatch ( $Z_{0}$ is the free space impedance).

\section{Conclusion}

In this paper, the concept of parallel-plates waveguide as an RF Wien filter has been shown. The required coupling between the electric and magnetic fields has been inherited from Maxwell equations. The orthogonality and homogeneity required for EDM measurements has been simulatively elaborated.

\section{References}

[1] V. Bargmann, Louis Michel, and V. L. Telegdi. Precession of the polarization of particles moving in a homogeneous electromagnetic field. Phys. Rev. Lett., 2:435-436, May 1959.

[2] Takeshi Fukuyama and Alexander J. Silenko. Derivation of generalized thomas-bargmann-michel-telegdi equation for a particle with electric dipole moment. International Journal of Modern Physics A, 28(29):1350147, 2013.

[3] Peter J. Mohr, Barry N. Taylor, and David B. Newell. Codata recommended values of the fundamental physical constants: 2010*. Rev. Mod. Phys., 84:1527-1605, Nov 2012.

[4] William M. Morse, Yuri F. Orlov, and Yannis K. Semertzidis. rf wien filter in an electric dipole moment storage ring: The "partially frozen spin" effect. Phys. Rev. ST Accel. Beams, 16:114001, Nov 2013.

[5] D.M. Pozar. Microwave Engineering. Wiley, 2004.

[6] Publication under preparation. Intended for the Journal of Nuclear Instruments and Methods in Physics Research Section A: Accelerators, Spectrometers, Detectors and Associated Equipment, 2016. 